\title{
Geodynamical value of historical geodetic measurements: A theoretical analysis
}

\author{
Peiliang $\mathrm{Xu}^{1}$, Seiichi Shimada ${ }^{2}$, Yoichiro Fujii ${ }^{3}$, and Torao Tanaka ${ }^{4}$ \\ ${ }^{1}$ Disaster Prevention Research Institute, Kyoto University at Uji, Uji, Kyoto 611-0011, Japan \\ ${ }^{2}$ Natl. Res. Inst. for Earth Sc. and Disaster Prevention, Tennodai 3-1, Tsukuba, Ibaraki 305-0006, Japan \\ ${ }^{3}$ Nippo Co. Ltd., 1-8-7 Kodai, Miyamae-ku, Kawasaki 216, Japan \\ ${ }^{4}$ Meijo University, Nagoya, Japan
}

(Received December 6, 1999; Revised August 10, 2000; Accepted September 12, 2000)

\begin{abstract}
Historical geodetic measurements have been used to infer on the displacement and strain states locally or regionally. They are also often used to invert for other geophysical parameters. However, historical geodetic measurements have been known to contain significant scaling and orientation errors, which may even be different in different parts of a network. These significant error sources may result in producing a wrong (or at least, a misleading) displacement or strain field. When such a displacement or strain field is further used to invert certain geophysical parameters, mis-interpretations may be expected. Thus, in this paper, we will perform a theoretical analysis to answer the following three questions: (i) are displacements obtainable from historical geodetic data? (ii) are strains obtainable from historical geodetic data? and (iii) what geodynamical value do historical geodetic data have?
\end{abstract}

\section{Introduction}

Although modern space geodetic techniques are very precise for a variety of geodynamical purposes, they were routinely operational only in the last one or two decades. In order to understand immediate or long term geodynamical processes locally or regionally, we have to deal with historical geodetic data (see, e.g. Bibby, 1975; Brunner et al., 1981; Frank, 1966; Fujii and Nakane, 1983; Gu and Prescott, 1986; Komaki, 1993a, b; Prescott, 1981; Savage and Burford, 1970; Savage et al., 1981a, b; Shen et al., 1996; Thatcher, 1979; Welsch, 1983). In Japan, the first order national triangulation network was started in 1882 and completed around 1909, which was then readjusted and the results were reported in Komaki (1985). These historical geodetic measurements have played an important role in understanding, inferring and inverting the geodynamical processes (earthquakes, for example) in Japan. However, one should exercise great care in using and interpreting the geodynamical information derived from the historical geodetic measurements geophysically. The historical geodetic measurements were inherently contaminated by three significant problems: (i) unknown scalings and orientations can be different in different parts of a network; (ii) unknown scalings and orientations can be different at different epochs of geodetic measurements; and (iii) the historical geodetic data are generally of poor accuracy. For the first order triangulation networks, different scaling errors of up to $37 \mathrm{ppm}$ have been found by directly comparing the baselines of the 1883 triangulation network with modern precise distance measurements (see, e.g. Komaki, 1985; Nakane, 1991). A scaling error at this level can certainly not result in any meaningful or robust estimates of strains. In

Copy right (C) The Society of Geomagnetism and Earth, Planetary and Space Sciences (SGEPSS); The Seismological Society of Japan; The Volcanological Society of Japan; The Geodetic Society of Japan; The Japanese Society for Planetary Sciences. this paper, for simplicity, we only discuss a simplified case by assuming: (i) that a set of datum conditions is valid for the whole network but unknown; and (ii) that different epochs of measurements have a different set of unknown datum conditions. In other words, there exists no referential connection between any two epochs of measurements.

Given two epochs of geodetic measurements, the displacements of the network points have been almost always computed by

$$
\nabla \mathbf{X}_{g}=\mathbf{N}_{2}^{+} \mathbf{A}_{2}^{T} \mathbf{P}_{2} \mathbf{y}_{2}-\mathbf{N}_{1}^{+} \mathbf{A}_{1}^{T} \mathbf{P}_{1} \mathbf{y}_{1}
$$

where $\mathbf{N}_{1}=\mathbf{A}_{1}^{T} \mathbf{P}_{1} \mathbf{A}_{1}$ and $\mathbf{N}_{2}=-\mathbf{A}_{2}^{T} \mathbf{P}_{2} \mathbf{A}_{2}$ are the normal matrices at epochs 1 and 2. + stands for the Moore-Penrose inverse of a matrix. Other notations will be explained later in (3). Taking the problems of historical geodetic measurements described in the above, one has a right reason to ask: (i) how accurate and reliable the displacements (1) are; and (ii) if the historical geodetic measurements are contaminated by a set of unknown but significant datum conditions, what the formula (1) actually produces? Does it produce a physically meaningful displacement field? In this paper, we are only concerned with the second question. Our answer to this question is that (1) does not mathematically produce a physical displacement field. This explains why we used the notation $\nabla \mathbf{X}_{g}$ instead of a commonly used notation for a displacement vector. If (1) is not a displacement field from historical geodetic data, then any use of it for inferring on geodynamical processes or inverting geodynamical parameters may result in significant implications.

Very often, one will then use the results of (1) to compute the strain/stress field in the network area. Instead of first computing the solution for each epoch and then the coordinate differences of the common network points, one can 
directly compute the coordinate differences as in (1) from the measurements of two epochs (see, e.g. Yu and Segall, 1996). However, these methods are mathematically essentially equivalent, which can be readily proved by using the theorem of equivalent measurements in linear models. Basing our paper on (1) thus does not lose any generality. Since a geodetic network can only provide pointwise values, a certain approximation has to be assumed in order to make the computation of strain/stress possible. Generally, a first order linear approximation is adopted. Thus the strains in the network have been computed by using the following system of linear equations:

$$
\nabla \mathbf{X}_{g}=\mathbf{E}_{s} \mathbf{X}-\Omega \mathbf{X}+\mathbf{u}_{0}
$$

where $\mathbf{E}_{s}$ is the strain tensor to be determined. In the threedimensional (3D) case, $\mathbf{E}_{s}$ has six independent elements. $\Omega$ is the anti-symmetric tensor, which has three independent elements and describes the rigid rotation of the network. $\mathbf{u}_{0}$ is a constant vector and describes the rigid movement of the whole network. If $\nabla \mathbf{X}_{g}$ of (1) is not a displacement field from historical geodetic data, as was pointed out earlier, then we also have a good reason to ask whether the computed $\mathbf{E}_{s}$ is a physical strain tensor. The answer to this question is theoretically negative, unfortunately. It is noted that a less general and not optimal method to compute shear from repeated geodetic measurements was proposed by Frank (1966).

So far, we have outlined the three major motivations for this paper and provided our answers to some posed questions without a solid foundation. Before we outline the organizations of this paper, we expect that the reader may ask now that if displacements and strains could not be derived from historical geodetic data, what geodynamical information might be obtained. Indeed, although historical geodetic data cannot provide displacements and strains, they are still valuable since they do contain valuable geodynamical information. The paper is thus organized as follows. Section 2 will develop a new observation model for historical geodetic data. This model will be used in Section 3 to explain: (i) why (1) is not a displacement field; (ii) why (2) is not a physical strain field; and (iii) what geodynamical value historical geodetic measurements may have. For some relevant analysis, the reader is referred to Dermanis (1981, 1985, 1994), Bibby (1982) and Chrzanowski et al. (1983).

\section{Models for Geometric Geodetic Measurements}

Geometric geodetic observations, such as distance, direction and angle measurements, have been used to establish local, national and continental control networks. The coordinates of the benchmarks in a control network are referred to a certain set of starting computation data, i.e. so-called geodetic datum in geodesy, which is mathematically arbitrary. A geodetic datum usually includes the coordinates of a fixed benchmark, a fixed distant length, and orientations of the network, and uniquely determines the positions of all the other control points of the network. If the selected geodetic datum is supposed to remain unchanged over time, i.e. no movement at the referred fixed point and no changes in the selected distance and orientations over time, then geodetic techniques can be used to derive the displacements of all the other stations of the network, which was exactly a common practice before the free network adjustment method was invented by Meissl $(1962,1965,1969)$.

If repeated geometric geodetic observations are used to detect geodynamical deformation, in particular, of long term nature, it is theoretically not acceptable that a geodynamical process would have only displaced all the benchmarks except for the pre-selected geodetic datum data. This argument invalidates the classical adjustment method with a fixed geodetic datum for retrieval of crustal deformation information. In order to overcome the deficiency of the classical adjustment theory, Meissl (1962) first developed a new theory to process geometric geodetic observations, which is well known as free network adjustment. Now let us assume that there exists no fixed geodetic datum for the geodetic network. In other words, assume that all the benchmarks of a geodetic network are located on a deformable body (part of the Earth surface, for example) in the language of geodynamics. Then geometric geodetic observations are linearized with respect to a certain pre-selected geodetic coordinate system and can be written as:

$$
\left.\begin{array}{l}
E(\mathbf{y})=\mathbf{A} \Delta \mathbf{X}, \mathbf{y}=\mathbf{A} \Delta \mathbf{X}+\boldsymbol{\varepsilon} \\
D(\mathbf{y})=\mathbf{P}^{-1} \sigma^{2}
\end{array}\right\},
$$

where $E(\cdot)$ stands for the operator of mathematical expectation, $\mathbf{y}$ is the difference between the observation vector and its approximation. $\mathbf{A}$ is the design matrix of order $(n \times t)$ with rank $r(<t) . \Delta \mathbf{X}$ is the coordinate correction vector in the initial (arbitrary) coordinate system. $\boldsymbol{\varepsilon}$ is the observation error vector with the weight matrix $\mathbf{P}$ and $\sigma^{2}$ the unknown variance component of unit weight.

The model (3) has been proved to be valid only within the initially (arbitrarily) pre-selected geodetic coordinate system (Xu, 1994, 1997). If a set of geodetically meaningful datum conditions is applied to (3), or equivalently, if a generalized inverse of the matrix $\mathbf{A}$ is used, one can derive a general solution, say, $\mathbf{X}_{i s}$, where the subscript $i s$ annotates the initially pre-selected coordinate system to the solution of the model (3). It is well known that triangulation and trilateration are invariant with respect to rotation and translation (see e.g. Baarda, 1973; Grafarend and Schaffrin, 1976; Dermanis and Grafarend, 1981; Bibby, 1982; Segall and Matthews, 1988). However, the general solution $\mathbf{X}_{i s}$ to (3) does not reflect this important observation of invariance. This is obviously not theoretically acceptable, and should indicate that the starting model (3) for free network adjustment is not sufficiently general mathematically. The fact that a free network adjustment model (3) for geometric geodetic observations loses generality might have been hinted at in the literature (see e.g. Baarda, 1973; Dermanis and Grafarend, 1981; Bibby, 1982; Segall and Matthews, 1988). No implications of such a loss of generality on geodynamical practice such as computing (so-called) displacements and strains from geodetic observations have been investigated, which partly motivates this paper. A most general model for free network adjustment was only recently proposed by Xu $(1993,1994,1997)$ 
however, and is rewritten as follows:

$$
\left.\begin{array}{l}
E(\mathbf{y})=\frac{1}{\lambda} \mathbf{A} \mathbf{U}^{-1} \Delta \mathbf{X}_{A R} \\
\mathbf{y}=\frac{1}{\lambda} \mathbf{A} \mathbf{U}^{-1} \Delta \mathbf{X}_{A R}+\boldsymbol{\varepsilon} \\
D(\mathbf{y})=\mathbf{P}^{-1} \sigma^{2}
\end{array}\right\},
$$

where $\Delta \mathbf{X}_{A R}$ is the coordinate correction vector within a general Euclidean three-dimensional coordinate system $S_{A R} . \lambda$ is any positive number and represents a scaling for a particular solution to the model (4). $\mathbf{A}$ and $\mathbf{P}$ are defined as in (3). $\mathbf{U}$ is a rotation matrix and is given by

$$
\begin{gathered}
\mathbf{U}=\left(\mathbf{I} \otimes \mathbf{U}_{0}\right), \\
\mathbf{U}_{0}=\mathbf{U}_{z} \mathbf{U}_{y} \mathbf{U}_{x},
\end{gathered}
$$

where $\otimes$ is the operation of Kronecker product of matrices, and $\mathbf{U}_{x}, \mathbf{U}_{y}$ and $\mathbf{U}_{z}$ respectively defined by

$$
\begin{aligned}
\mathbf{U}_{x} & =\left(\begin{array}{ccc}
1 & 0 & 0 \\
0 & \cos \theta_{x} & \sin \theta_{x} \\
0 & -\sin \theta_{x} & \cos \theta_{x}
\end{array}\right), \\
\mathbf{U}_{y} & =\left(\begin{array}{ccc}
\cos \theta_{y} & 0 & \sin \theta_{y} \\
0 & 1 & 0 \\
-\sin \theta_{y} & 0 & \cos \theta_{y}
\end{array}\right), \\
\mathbf{U}_{z} & =\left(\begin{array}{ccc}
\cos \theta_{z} & \sin \theta_{z} & 0 \\
-\sin \theta_{z} & \cos \theta_{z} & 0 \\
0 & 0 & 1
\end{array}\right) .
\end{aligned}
$$

Here $\theta_{x}, \theta_{y}$ and $\theta_{z}$ are three rotation angles. The model (4) is general for triangulation networks. For trilateration networks and/or mixing of triangulation and trilateration networks, the scaling parameter $\lambda$ is fixed and can often take on unity.

The total geometric information contained in the geometric geodetic observations $\mathbf{y}$ can be summarized in the following theorem.

Theorem 1 (Xu, 1997): If $\mathbf{N}^{-}$is a generalized inverse of the normal matrix $\mathbf{N}=\left(\mathbf{A}^{T} \mathbf{P A}\right)$, then the geometric geodetic observation model (4) has a geodetically general solution of coordinates:

$$
\mathbf{X}_{g}=\lambda \mathbf{U} \mathbf{N}^{-} \mathbf{A}^{T} \mathbf{P y}+\lambda \mathbf{U} \mathbf{X}_{0}+\left(\mathbf{1} \otimes \mathbf{Z}_{t}\right),
$$

where $\mathbf{X}_{g}$ is the coordinate representation of the geometric geodetic observations, $\mathbf{X}_{0}$ is the approximate values of coordinates in the initially selected coordinate system, $\mathbf{1}$ is a vector with all its elements equal to unity. $\mathbf{Z}_{t}=\left(z_{t x}, z_{t y}, z_{t z}\right)^{T}$, three free translation elements.

The solution (5) is a general coordinate representation of the total geometric information in the observations within any Euclidean coordinate system. As a simple consequence, the least squares (LS) minimum norm (LSMN) or MoorePenrose generalized inverse solution to the model (3) or (4) is a particular solution of (5), although the LSMN solution was shown to result in the minimum trace among the generalized inverses of ( $\left.\mathbf{A}^{T} \mathbf{P A}\right)$. From the point of view of invariant geometric information, the LSMN solution is not better nor worse than any other particular one that can be derived from (5).

The solution (5) is most general for any geometric geodetic observations. We will show two examples of (5) in the following. If all the geometric observations are of levelling type, the heights of the benchmarks in a levelling network can be written from (5) as:

$$
\mathbf{H}_{g}=\mathbf{N}^{-} \mathbf{A}^{T} \mathbf{P y}+\mathbf{H}_{0}+z_{t h} \mathbf{1},
$$

if the scaling is assumed fixed. Here $\mathbf{H}_{g}$ is the height vector, $\mathbf{H}_{0}$ is the initially selected height vector, and $z_{t h}$ is an arbitrary constant. For GPS-derived baseline vectors, the solution (5) becomes:

$$
\mathbf{X}_{g}=\mathbf{N}^{-} \mathbf{A}^{T} \mathbf{P y}+\mathbf{X}_{0}+\left(\mathbf{1} \otimes \mathbf{Z}_{t}\right)
$$

if the GPS-derived baseline vectors are assumed to have a fixed scaling and orientations.

\section{Geodynamical Information in Historical Geodetic Measurements}

In this section, we assume that all the network stations are located on a deformable body and are all influenced by some geodynamical processes in an immediate or long term. We also assume that each epoch of measurements corresponds to one set of unknown datum conditions. These assumptions are justified, since the errors of scalings and orientations in historical geodetic data are generally very large. Based on these conditions, we would like to investigate three issues: (i) whether displacements can or cannot be obtained from historical geodetic data; (ii) whether strains can or cannot be obtained from historical geodetic data; and (iii) what geodynamical value historical geodetic data may have.

\subsection{Are displacements derivable from historical geode- tic data}

Given two epochs of historical geodetic data as modeled by (4), we have two sets of unknown datum conditions accordingly. The coordinate difference, or more precisely, the difference of the two general solutions, can be computed by using (5) as follows:

$$
\begin{aligned}
\nabla \mathbf{X}_{g}= & \mathbf{X}_{g 2}-\mathbf{X}_{g 1} \\
= & \lambda_{2} \mathbf{U}_{2} \mathbf{N}_{2 I I}^{-} \mathbf{A}_{2}^{T} \mathbf{P}_{2} \mathbf{y}_{2}-\lambda_{1} \mathbf{U}_{1} \mathbf{N}_{1 I}^{-} \mathbf{A}_{1}^{T} \mathbf{P}_{1} \mathbf{y}_{1} \\
& +\lambda_{2} \mathbf{U}_{2} \mathbf{X}_{02}-\lambda_{1} \mathbf{U}_{1} \mathbf{X}_{01}+\left(\mathbf{1} \otimes \mathbf{Z}_{t 12}\right),
\end{aligned}
$$

where $\mathbf{Z}_{t 12}=\mathbf{Z}_{t_{2}}-\mathbf{Z}_{t_{1}}$. Since (5) represents the measured data in the manner of an arbitrarily selected coordinate system, $\nabla \mathbf{X}_{g}$ can also be properly called the difference between the two representations of coordinates at the time epochs $t_{1}$ and $t_{2}$.

Due to the space limit, we will not be able to discuss (6) in detail. Instead, we will simply summarize some major observations on (6). The interested reader is asked to refer to Xu et al. (2000) for more details.

Important observations on (6) are listed as follows:

- $\nabla \mathbf{X}_{g}$ contains all the geometric information in $\mathbf{y}_{1}$ and $\mathbf{y}_{2}$, since (5) fully represents all the information for each epoch of measurements; 
- $\nabla \mathbf{X}_{g}$ is the mix of $\mathbf{y}_{1}, \mathbf{y}_{2}$ and all the datum-related data such as $\lambda, \mathbf{U}$ and (different) generalized inverses of the normal matrices;

- because of Observation 2, $\nabla \mathbf{X}_{g}$ is not a displacement field;

- the concept of relative displacement is not valid with historical geodetic data either, because

1. historical geodetic data are inherently influenced by different (unknown but significant) scalings and orientations in different parts of the network; and

2. even if the two sets of $\lambda, \mathbf{U}$ and $\mathbf{Z}_{t}$ are identical, different types of generalized inverses are mathematically applicable and equivalent in representing the geodetic data.

From these observations, we can readily conclude that the concept of displacement does not exist here with problematic historical geodetic data for detecting a geodynamical process that affect all the network stations in a long term. In this case, $\nabla \mathbf{X}_{g}$ may be more properly called misplacement (coined by Prof. E. Grafarend during one of the discussions with PX on geodetic deformation analysis in his Stuttgart office). Therefore, use of (6) must be exercised with great care, and any attempt to explain the results from using (6) geophysically is not without doubt. In fact, by assigning different datum data to (6), one can obtain up to an infinite number of displacement fields. It is expected that if a particular solution of (6), or so called displacement field, is used to invert for some geophysical parameters, then the inverted parameters are not free from the datum conditions. In other words, one can also obtain up to an infinite number of geophysical parameter sets. Which set of the inverted parameters is correct? This question cannot be answered from geometric geodetic data, unfortunately. However, we have to note that it is still possible to detect deformation or displacements of network points for a suddenly occurring, localized event. In this situation, we can safely assume that the two epochs of geodetic data share the same, determinable datum conditions.

\subsection{Are strains derivable from historic geodetic data}

A geodetic network can, at the very best, provide discrete deformation information in the network area. In order to infer on the strain state from geodetic data, a certain assumption on the deformation state has to be made. Very often, the following linear approximation of a $3 \mathrm{D}$ displacement field has been used, namely,

$$
\mathbf{u}=\mathbf{E}_{s} \mathbf{X}-\Omega \mathbf{X}+\mathbf{u}_{0},
$$

where $\mathbf{u}$ is the displacement vector at the point $\mathbf{X}, \mathbf{E}_{s}$ is the strain tensor, $\Omega$ is the anti-symmetric tensor, and $\mathbf{u}_{0}$ is a constant vector.

Collecting (7) at all the network points and replacing the coordinate components of matrices $\mathbf{B}$ and $\mathbf{C}$ with $\mathbf{X}_{1}$, we have the system of equations:

$$
\mathbf{u}_{s}=\mathbf{B}_{s} \mathbf{e}_{s}-\mathbf{C}_{s} \boldsymbol{\omega}+\left(\mathbf{1} \otimes \mathbf{u}_{0}\right)+\boldsymbol{\epsilon}_{s},
$$

for computing the strains by the least squares method, where

$$
\begin{aligned}
& \mathbf{u}_{s}=\nabla \mathbf{X}_{g}=\mathbf{X}_{g 2}-\mathbf{X}_{g 1} \\
& =\lambda_{2} \mathbf{U}_{2} \mathbf{N}_{2 I I}^{-} \mathbf{A}_{2}^{T} \mathbf{P}_{2} \mathbf{y}_{2}-\lambda_{1} \mathbf{U}_{1} \mathbf{N}_{1 I}^{-} \mathbf{A}_{1}^{T} \mathbf{P}_{1} \mathbf{y}_{1} \\
& +\lambda_{2} \mathbf{U}_{2} \mathbf{X}_{02}-\lambda_{1} \mathbf{U}_{1} \mathbf{X}_{01}+\left(\mathbf{1} \otimes \mathbf{Z}_{t 12}\right) \text {, } \\
& \mathbf{B}_{s}=\left[\mathbf{B}_{1}^{T}, \mathbf{B}_{2}^{T}, \ldots, \mathbf{B}_{t}^{T}\right]^{T}, \\
& \mathbf{C}_{s}=\left[\mathbf{C}_{1}^{T}, \mathbf{C}_{2}^{T}, \ldots, \mathbf{C}_{t}^{T}\right]^{T}, \\
& \mathbf{B}_{i}=\left[\begin{array}{cccccc}
x_{i} & y_{i} & z_{i} & 0 & 0 & 0 \\
0 & x_{i} & 0 & y_{i} & z_{i} & 0 \\
0 & 0 & x_{i} & 0 & y_{i} & z_{i}
\end{array}\right] \text {, } \\
& \mathbf{C}_{i}=\left[\begin{array}{rrr}
0 & -z_{i} & y_{i} \\
z_{i} & 0 & -x_{i} \\
-y_{i} & x_{i} & 0
\end{array}\right],
\end{aligned}
$$

and

$$
\boldsymbol{\epsilon}_{s}=\left[\boldsymbol{\epsilon}_{1}^{T}, \boldsymbol{\epsilon}_{2}^{T}, \ldots, \boldsymbol{\epsilon}_{t}^{T}\right]^{T} .
$$

Again, due to the space limit, we can only summarize some major observations on (8) as follows:

- $\mathbf{u}_{0}$ cannot be separated from $\mathbf{Z}_{t 12}$, and thus cannot be determined;

- $\mathbf{u}_{s}$ is the mix of $\mathbf{y}_{1}, \mathbf{y}_{2}$ and all the datum-related data such as $\lambda, \mathbf{U}$ and (different) generalized inverses of the normal matrices;

- because of Observation 2, $\mathbf{E}_{s}$ cannot be uniquely determined from problematic historical geodetic data. If all the issues in Observation 2 are incorrectly eliminated, for instance, by simply assigning some datum and referential numbers to the corresponding formulas, as was exactly done in Bibby (1982) (though implicitly and likely without the knowledge of Bibby and others) and the relevant literature, one obtains some numbers for the tensor $\mathbf{E}_{s}$. In fact, when these authors claim the unbiasedness of the strain estimate, they implicitly assume that there exists a known connection of the referentials between two epochs of measurements. This implicit assumption becomes obvious in the light of the new general model (4) and contradicts with one of the basic starting points of free adjustment, i.e. no known referential connection. After eliminating this assumption from Bibby (1982), it becomes evident from the formulae in this Subsection that the unbiasedness result by Bibby (1982) is not correct in general. Therefore these numbers cannot be said to be a strain field physically, and certainly cannot be unbiased under the framework of free adjustment.

In order not to give the reader an impression that we cannot compute any physical quantities from geometric geodetic data, we would like to point out that if scaling and orientation errors are sufficiently small, the shear strain is almost invariant or can be almost uniquely determined. For more details, the reader is referred again to $\mathrm{Xu}$ et al. (2000). We also like to note that geodetic data are indeed able to produce the strain field if they are collected right before and after some localized events. In this case, we can safely assume 
that most of the network points are not influenced by such localized events and can be used to construct the required datum conditions.

\subsection{What are derivable from historical geodetic data}

Left-multiplying the rotation matrix $\mathbf{U}_{1}^{T}$ and then the design matrix $\mathbf{A}_{1}$ to both sides of (8), we obtain:

$$
\mathbf{A}_{1} \mathbf{U}_{1}^{T} \mathbf{u}_{s}=\mathbf{A}_{1} \mathbf{U}_{1}^{T} \mathbf{B}_{s} \mathbf{e}_{s}-\mathbf{A}_{1} \mathbf{U}_{1}^{T} \mathbf{C}_{s} \boldsymbol{\omega}+\mathbf{A}_{1}\left(\mathbf{1} \otimes \mathbf{u}_{0}\right)+\mathbf{A}_{1} \mathbf{U}_{1}^{T} \boldsymbol{\epsilon}_{s}
$$

or equivalently,

$$
\begin{aligned}
\mathbf{e}_{s u}= & {\left[\mathbf{B}_{s 0}^{T} \mathbf{A}_{1}^{T} \mathbf{Q}_{\epsilon}^{-} \mathbf{A}_{1} \mathbf{B}_{s 0}\right]^{-1} \mathbf{B}_{s 0}^{T} \mathbf{A}_{1}^{T} \mathbf{Q}_{\epsilon}^{-} } \\
& \cdot\left(\kappa \mathbf{F}\left(\boldsymbol{\theta}_{12}\right) \mathbf{I}_{u 2}-\mathbf{I}_{u 1}\right),
\end{aligned}
$$

(see, Xu et al., 2000), where $\mathbf{Q}_{\epsilon}$ is the variance-covariance matrix,

$$
\begin{gathered}
\mathbf{I}_{u 2}=\mathbf{A}_{1} \mathbf{N}_{2 I I}^{-} \mathbf{A}_{2}^{T} \mathbf{P}_{2} \mathbf{y}_{2}, \\
\mathbf{I}_{u 1}=\mathbf{A}_{1} \mathbf{N}_{1 I}^{-} \mathbf{A}_{1}^{T} \mathbf{P}_{1} \mathbf{y}_{1}, \\
\kappa=\lambda_{2} / \lambda_{1}, \\
\mathbf{e}_{s u}=v\left(\mathbf{U}_{0}^{T} \mathbf{E}_{s} \mathbf{U}_{0}\right),
\end{gathered}
$$

$\mathbf{F}\left(\boldsymbol{\theta}_{12}\right)$ is a matrix of the new rotations that actually represent the relative rotations of $\mathbf{U}_{1}^{T} \mathbf{U}_{2}$.

The space limit forces us to list some major observations on (9) as follows:

- the six components of the strain tensor are represented by three unknown relative rotations. Thus only three functional relationships of the strain components may be derivable;

- since the relative scaling $\kappa$ is unknown, the absolute magnitidues of the strain components cannot be determined either. Thus, at most, only three relations of relative tensor components could be uniquely determined.

- also note that the rigid rotation and translation motions are automatically cancelled out in the derivation of (9). Thus they definitely cannot be determined from historic geodetic data.

For more details, the reader is referred to $\mathrm{Xu}$ et al. (2000).

\section{References}

Baarda, W., S-Transformation and Criterion Matrices, Neth. Geod. Comm. Publ. Geod., New Series, Vol. 5, No. 1, 1973.

Bibby, H. M., Crustal strain from triangulation in Marlborough, New Zealand, Tectonophys., 29, 529-540, 1975.

Bibby, H. M., Unbiased estimate of strain from triangulation data using the method of simultaneous reduction, Tectonophys., 82, 161-174, 1982.

Brunner, F. K., R. Coleman, and B. Hirsch, A comparison of computation methods for crustal strains from geodetic measurements, Tectonophys., 71, 281-298, 1981.

Chrzanowski, A., Y. Q. Chen, and J. M. Secord, On the strain analysis of tectonic movements using fault crossing geodetic surveys, Tectonophys., 97, 297-315, 1983.

Dermanis, A., Geodetic estimability of crustal deformation parameters, Quaterniones Geod., 2, 159-169, 1981.

Dermanis, A., The role of frame definitions in the geodetic determination of crustal deformation parameters, Bull. Géod., 59, 247-274, 1985.
Dermanis, A., A method for the determination of crustal deformation parameters and their accuracy from distances, J. Geod. Soc. Japan, 40, 17-32, 1994.

Dermanis, A. and E. Grafarend, Estimability analysis of geodetic, astrometric and geodynamical quantities in very long baseline interferometry, Geophys. J. R. astr. Soc., 64, 31-64, 1981.

Frank, F. C., Deduction of earth strains from survey data, Bull. Seismol. Soc. Am., 56, 35-42, 1966.

Fujii, Y. and K. Nakane, Horizontal crustal movements in the Kanto-Tokai district, Japan, as deduced from geodetic data, Tectonophys., 97, 115$140,1983$.

Grafarend, E. and B. Schaffrin, Equivalence of estimable quantities and invariance in geodetic networks, Z. Verm., 101, 485-491, 1976.

Gu, G. and W. H. Prescott, Discussion on displacement: Detection of crustal deformation, J. Geophys. Res., B91, 7439-7446, 1986.

Komaki, K., The readjustment of the Meiji first order triangulation network by the projection method, Bull. Geogr. Surv. Inst. Japan, 29, 1-45, 1985.

Komaki, K., Horizontal crustal movements revealed by geodetic measurements: On the methods for estimating displacement vectors, Bull. Geogr. Surv. Inst. Japan, 39, 1-41, 1993a.

Komaki, K., Horizontal crustal movements revealed by geodetic measurements: Applications of a new method for estimating displacement vectors, J. Geod. Soc. Japan, 39, 387-410, 1993 b.

Meissl, P., Die innere Genauigkeit eines Punkthaufens, Öster. Z. Verm., 50, 159-165, 186-194, 1962.

Meissl, P., Über die innere Genauigkeit dreidimensionaler Punkthaufen, $Z$. Verm., 90, 109-118, 1965.

Meissl, P., Zusammenfassung und Ausbau der inneren Fehlertheorie eines Punkthaufens, in Beiträge zur Theorie der Geodätischen Netze im Raum, DGK, A61, pp. 8-21, edited by R. Rinner, K. Killian, and P. Meissl, German Geod. Comm., Munich, 1969.

Nakane, K., Scale accuracy of geodetic network in Japan, Bull. Geogr. Surv. Inst. Japan, 36, 1-19, 1991.

Prescott, W. H., The determination of displacement fields from geodetic data along a strike slip fault, J. Geophys. Res., B86, 6067-6072, 1981.

Savage, J. C. and R. O. Burford, Accumulation of tectonic strain in California, Bull. Seismol. Soc. Am., 60, 1877-1896, 1970.

Savage, J. C., M. Lisowski, and W. H. Prescott, Geodetic strain measurements in Washington, J. Geophys. Res., B86, 4929-4940, 1981a.

Savage, J. C., W. H. Prescott, M. Lisowski, and N. E. King, Strain accumulation in southern California, 1970-1980, J. Geophys. Res., B86, 6991-7001, 1981b.

Segall, P. and M. V. Matthews, Displacement calculation from geodetic data and the testing of geophysical deformation models, J. Geophys. Res., B93, 14954-14966, 1988.

Shen, Z. K., D. Jackson, and B. Ge, Crustal deformation across and beyond the Los Angeles basin from geodetic measurements, J. Geophys. Res., B101, 27957-27980, 1996.

Thatcher, W., Horizontal crustal deformation from historic geodetic measurements in southern California, J. Geophys. Res., B84, 2351-2370, 1979.

Welsch, W., Finite element analysis of strain patterns from geodetic observations across a plate margin, Tectonophys., 97, 57-71, 1983.

$\mathrm{Xu}, \mathrm{P}$. L., Testability, datum definitions, coordinate systems and free net adjustment, Pres. at 8th Int. Symp. on Recent Crustal Movement, Kobe, Japan, Dec. 6-10, 1993, 1993.

$\mathrm{Xu}, \mathrm{P}$. L., Testability and adjustment in free net models, J. Geod. Soc. Japan, Suppl. issue, 40, 315-320, 1994.

$\mathrm{Xu}, \mathrm{P}$. L., A general solution in geodetic nonlinear rank-defect models, Boll. Geod. Sc. Affini, 56, 1-25, 1997.

Xu, P. L., S. Shimada, Y. Fujii, and T. Tanaka, Invariant geodynamical information in geometric geodetic measurements, Geophys. J. Int., 142, 586-602, 2000.

Yu, E. and P. Segall, Slip in the 1868 Hayward earthquake from the analysis of historical triangulation data, J. Geophys. Res., B101, 16101-16118, 1996.

P. Xu (e-mail: pxu@rcep.dpri.kyoto-u.ac.jp), S. Shimada, Y. Fujii, and T. Tanaka 